\title{
Usage and Clinical Perspectives of Silicone Oil as a Therapeutic Adjunct in Hand Rehabilitation: A Survey of New Zealand and Australian Therapists
}

Gail Donaldson DipPhty, MPhty

Centre for Health, Activity, and Rehabilitation Research, School of Physiotherapy, University of Otago, Dunedin, New Zealand

Gillian M. Johnson MSc, PhD, FNZCP

Centre for Health, Activity, and Rehabilitation Research, School of Physiotherapy, University of Otago, Dunedin, New Zealand

Gisela Sole BSc(Physio), MSc(Med), PhD, FNZCP

Centre for Health, Activity, and Rehabilitation Research, School of Physiotherapy, University of Otago, Dunedin, New Zealand

Meredith Perry BPhty, MManipTh, PhD

Centre for Health, Activity, and Rehabilitation Research, School of Physiotherapy, University of Otago, Wellington, New Zealand

\section{ABSTRACT}

This study aimed to quantify current silicone oil usage by therapists in New Zealand and Australia, and gauge clinician perceptions regarding the therapeutic properties of silicone oil. To ascertain clinical beliefs, a questionnaire containing Likert scales was custom designed. Therapists attending a combined hand conference held in Melbourne, Australia, in 2013 were surveyed, with a 50.4\% response rate. One-quarter of therapists surveyed $(n=126)$ reported current usage of silicone oil. Therapists' perceptions were that silicone oil impacted positively on wound healing (Likert scale agreement score, 4.6/5) and finger movement, with less pain reported (Likert scale agreement score, 3.8/5). Silicone oil was used specifically after Dupuytren's palmar contracture release surgery. Non-use of silicone oil was mainly attributable to unavailability in the clinical setting. Results indicate that silicone oil is currently used and valued due to its pain-relieving and movement-promoting properties in combination with facilitation of wound healing.

Donaldson, G., Johnson, G. M., Sole, G. \& Perry, M. (2021). Usage and clinical perspectives of silicone oil as a therapeutic adjunct in hand rehabilitation: A survey of New Zealand and Australian therapists. New Zealand Journal of Physiotherapy, 49(1), 7-14. https://doi.org/10.15619/NZJP/49.1.02

Key Words: Hand, Pain, Physical Therapy, Silicone Oil, Wound Healing

\section{INTRODUCTION}

The hand, by virtue of its functional interaction with the environment, frequently sustains trauma that disrupts the integrity of the skin (Kwan et al., 2009). Traumatic hand injuries, such as burns, lacerations, and abrasions, are common and frequently require acute medical interventions and ongoing care to achieve wound healing (Lazarus et al., 1994). Substantial wounds of the hand are often challenging to manage, as the multiple joints, and underlying tissues and tendons require controlled motion during healing to prevent the formations of unwanted adhesions (Merritt, 1998). Concurrently, the outer surface of the hand needs to re-establish skin integrity as the wound closes, but still have sufficient laxity to allow all joints full motion (Yang et al., 2014).

Since the 1960s, medical grade silicone oil (SiO) has been proposed as a therapeutic adjunct for open hand wounds, burns, and post-operative hand rehabilitation (Helal et al., 1982; Spira et al., 1967). Medical grade SiO is clear and odourless, with a viscosity of 350 centistokes (cS), which is similar to olive oil. In comparison water, has a viscosity of $1 \mathrm{CS}$ at $20^{\circ} \mathrm{C}$ (Braley, 1970).
Anecdotal evidence suggests that SiO encourages early gains in finger motion with less associated pain (Helal et al., 1982; Spira et al., 1967) and increases the speed of wound healing, i.e., time taken to complete epithelisation (Weeder et al., 1967). Laboratory-based research has shown that SiO has antimicrobial properties, particularly against staphylococcal pathogens (Arici et al., 2016; Chrapek et al., 2012; Ozdamar et al., 1999). However, there is limited clinical research relating directly to hand wounds.

Despite historical case studies detailing the use of SiO for hand injuries (Helal et al., 1982; Maciejczyk, 1961; Spira et al., 1967; Weeder et al., 1967) and more recent ophthalmic studies (Dave et al., 2019), there has been no literature published during the last 18 years detailing the use of $\mathrm{SiO}$ in hand rehabilitation. In Australasia there are anecdotal reports of hand therapists using $\mathrm{SiO}$, but it is unclear whether therapists regularly use $\mathrm{SiO}$ as an adjunct in hand rehabilitation. The purpose of this paper was to clarify SiO use in New Zealand and Australia and, if used, to gauge clinician's perceptions about its properties and effectiveness. 


\section{METHODS}

This study is survey based, using a custom-designed questionnaire. A schematic overview of the study design is provided in Figure 1.

Questionnaire development

The University of Otago Human Ethics Committee granted ethical approval for this study (reference number D13/346). The questionnaire development included an initial literature search, an interview with two hand therapists, and a pilot test, from which five initial questions were identified:

1. Is SiO acceptable as an adjunct for open wound rehabilitation?

2. Are there adverse effects or risks associated with SiO usage?

3. What is the possible impact of $\mathrm{SiO}$ on pain experienced during exercise?

4. What impact does SiO have on finger range of motion?

5. What perceptions do therapists have regarding infection and wound healing with SiO usage?

The primary author (GD) conducted focus interviews with two hand therapists experienced in $\mathrm{SiO}$ use to gauge their opinion regarding the utility and overall scope of the questionnaire. Following these interviews, the cost of $\mathrm{SiO}$ and the impact on wound dressing changes were included in the questionnaire. Pilot testing on a convenience sample of five hand therapists during a regional hand therapy meeting determined minor formatting changes required to improve the questionnaire's clarity.

The silicone oil questionnaire

The final version of the questionnaire, entitled "The Silicone Oil in Hand Rehabilitation Questionnaire" (SiOQ) (Appendix A), contained 18 questions. Questions 1 to 6 entailed respondents' professional demographic and professional registration status data. Question 7 asked whether the respondent used SiO, followed by two open-ended questions asking therapists' reasons for using or not using SiO. Questions 10 to 16 were completed by users of SiO to examine professional viewpoints of SiO use relating to finger range of motion, pain levels, impact on dressing changes, adverse effects, and risks of infection. Respondents rated their agreement with the statements on a 5-point Likert scale, which was anchored by the reference points "disagree", "unsure" (centred), and "agree". Finally, questions 17 and 18 were open-ended and related to therapists' perceptions of the benefits and disadvantages of SiO.

\section{Administration of the SiOQ}

To qualify for inclusion in the SiOQ, participants had to be a hand therapist registered with either a New Zealand or Australian parent body; had to be an attendee at the combined conference of Hand Therapy New Zealand (HTNZ) and the Australian Hand Therapy Association (AHTA) that was held in Melbourne, Australia in October 2013; and had to provide formal consent.

In 2013, the collective membership of HTNZ and AHTA therapists was estimated to be 450 . Recruitment of conference attendees $(n=250)$ was via a poster in the registration area and announcements made at the conference. Respondents who completed hard copies of the participant information sheet, consent form, and SiOQ went into a draw to win an iPad mini.

Data analysis

Data were entered into Microsoft Excel for analysis. Respondent demographics and Likert scale responses were analysed using frequency counts. Likert response categories were ranked numerically and then multiplied by the frequencies. This weighted analysis (Norman, 2010) gave an overall combined score out of 5 points. This score, termed "level of agreement", reflected the respondent's viewpoint of each statement on the

\section{Figure 1}

Overview of Study Design

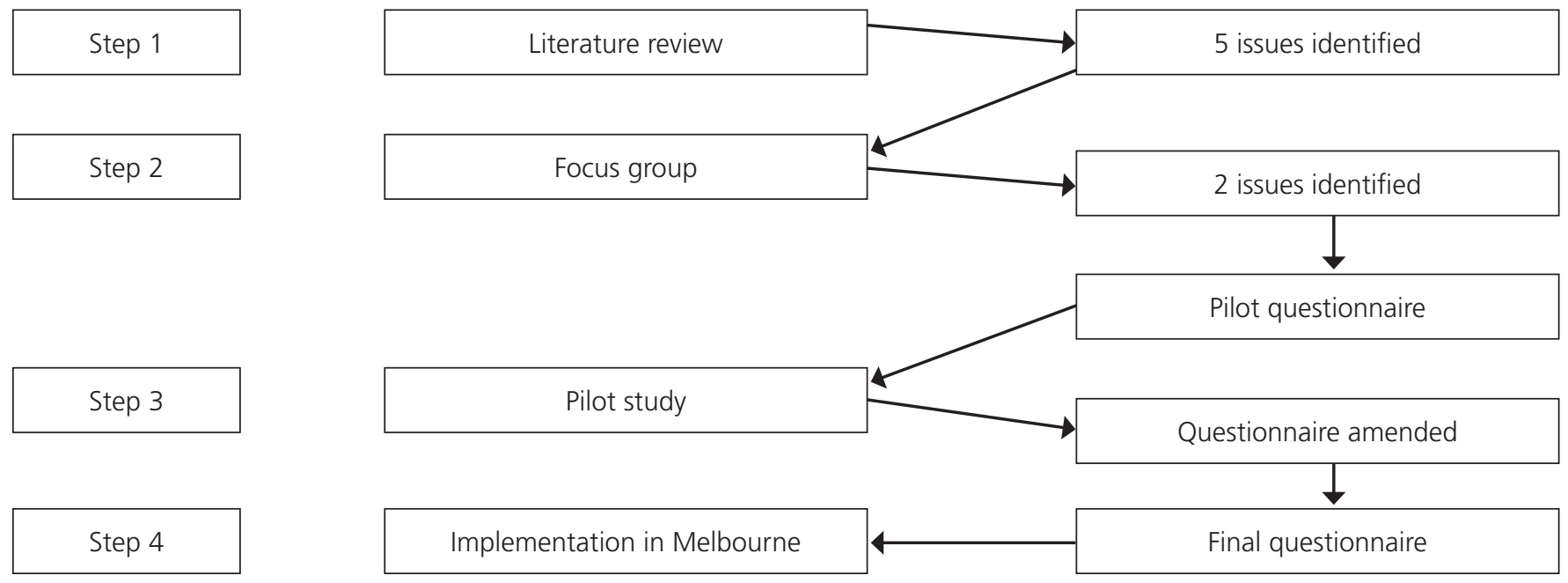


Likert scale. Zero agreement is reflected by a 0 score, whereas strong agreement has 5 points. Written responses to openended questions were grouped according to thematic content by GD, with frequency counts performed for reporting purposes.

\section{RESULTS}

\section{Demographics}

The response rate from the hand therapists attending the combined conference was $50.4 \%(n=126)$. All respondents were qualified therapists, who had either completed occupational therapy $(n=72)$ or physiotherapy $(n=51)$ training. All but three respondents provided hand therapy professional membership status and country of registration. The respondents reported a wide range of hand therapy experience (1-40 years), with a mean of 12 years $(S D=9)$. Of the respondents, 22 held postgraduate qualifications of either a master's degree or above, with three holding doctorates.

\section{SiO usage}

In regard to $\mathrm{SiO}$ usage, $43 \%(n=54 / 126)$ of respondents reported using $\mathrm{SiO}$ in their clinical practice. However, only $24 \%$ ( $n=30 / 126)$ currently used SiO. A third of respondents ( $n=$ $42 / 126$ ) were unaware of the existence of SiO as a therapeutic adjunct for hand therapy (Figure 2). The demographics of the SiO users $(n=54)$ compared to those who had never used SiO ( $n=72$ ) were similar (Table 1). A greater number of hand therapists with physiotherapy training $(62 \%, n=34 / 54)$ reported use of $\mathrm{SiO}$ in their clinical practice compared to those with an occupational therapy background $(37 \%, n=20 / 54)$.

\section{Agreement on clinical attributes of SiO}

The highest overall level of agreement for all respondents ( $n=$ 54) that had used SiO was that immersion was an acceptable and appropriate therapeutic adjunct for the management of open hand wounds (level of agreement score, 4.1/5). The second area of strong respondent agreement concerned the

\section{Figure 2}

Summary of Respondent Awareness and Usage of Silicone Oil
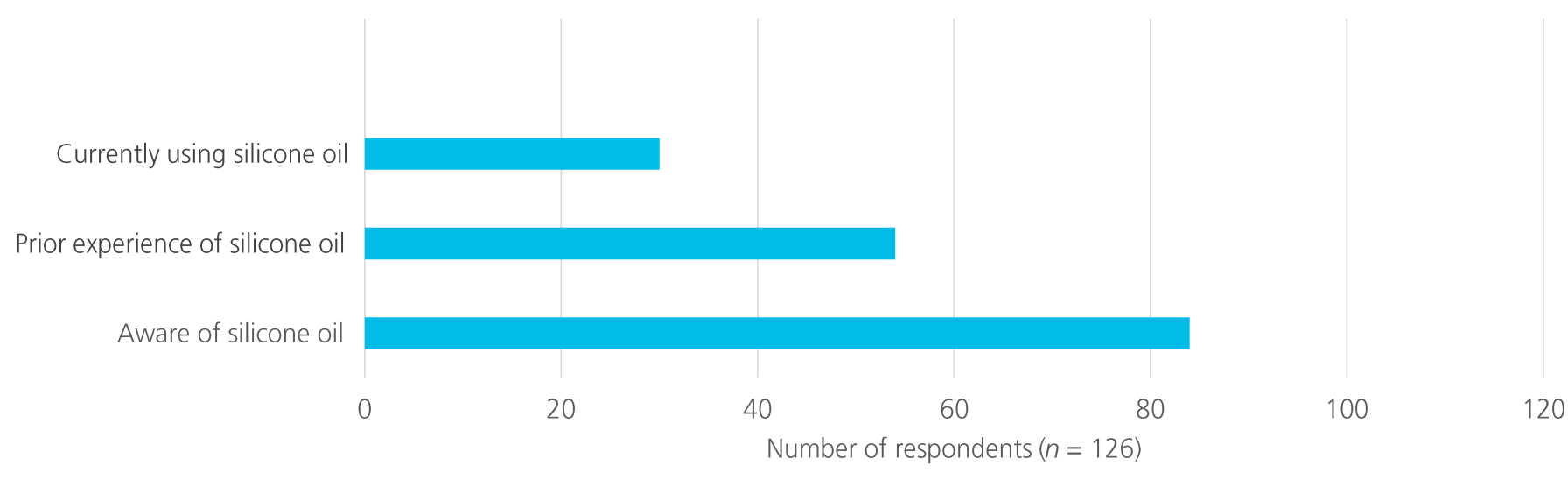

Table 1

Demographics of Respondents Separated by Silicone Oil Use

Total respondents in survey

$(n=126)$

Response rate (total 50.4\%)

Physiotherapist

Occupational therapist

Average years worked

Range of years worked

Private practice

Public practice

Master's degree or doctorate

New Zealand registered

Australian registered

Registration not stated
Silicone oil users

$$
(n=54)
$$

Never used silicone oil $(n=72)$

$\begin{array}{cc}43 \% & 57 \% \\ 34 & 17 \\ 20 & 55 \\ 14 & 11 \\ 1-40 & 0-32 \\ 41 & 48 \\ 13 & 24 \\ 11 & 11 \\ 30 & 4 \\ 10 & 32 \\ 14 & 36\end{array}$


positive impact of $\mathrm{SiO}$ on wound dressing changes, with the perception that dressing changes were atraumatic after $\mathrm{SiO}$ use (level of agreement score, 4.6/5). Respondents agreed that $\mathrm{SiO}$ allowed hand movement with less pain (level of agreement score, 3.8/5). The lowest level of agreement between SiO users (level of agreement score, 3.6/5) related to the risk of infection associated with SiO use (Table 2).

\section{Reasons for non-usage of SiO}

The most frequent reason for non-usage of SiO was its nonavailability in the clinic $(n=13)$ (Figure 3). Additional reasons included a lack of product knowledge combined with perceived limited clinical evidence for SiO use, and concerns over cleanliness and hygiene. Cost and the requirement for a doctor's referral were also reasons for nonuse. The free text responses regarding $\mathrm{SiO}$ disadvantages included responses about the "complex" and "messy" nature of the intervention, which has a "risk of spillage" (Appendix A, question 18). In addition, SiO was reported as "requiring a high level of cooperation and commitment from patients when used at home".
Reasons for use of SiO

Therapists reported SiO for specific use in the post-surgical management of Dupuytren's contracture release $(26 \%, n=$ 14) (Figure 4). Reasons for clinical use included "wound care" and "improved wound healing time", which together equalled $35 \%(n=19)$; gaining range of movement $(11 \%, n=6)$; at the surgeon's request $(9 \%, n=5)$; and for scar management $(9 \%, n=5)$. Respondents comments about perceived benefits included terms such as "soothing", "pain-free effect", and "less tightness when moving". The most cited observations related to the enhancement of wound healing: "faster wound healing" and "softer dead skin allowing for simpler debridement" (Appendix A, question 17).

\section{DISCUSSION}

A quarter of the therapists surveyed used $\mathrm{SiO}$, and strongly agreed that $\mathrm{SiO}$ was effective for open wound hand rehabilitation as it made movement less painful. Therapists stated that SiO was used specifically for post-surgical

\section{Table 2}

Level of Agreement Scores for Therapeutic Implications of Silicone Oil

\begin{tabular}{lcc}
\hline Therapeutic implications & \multicolumn{2}{c}{ Level of agreement ${ }^{\text {a }}$} \\
\cline { 2 - 3 } & $\begin{array}{c}\text { Current user of SiO } \\
(n=30)\end{array}$ & $\begin{array}{c}\text { Cused SiO } \\
(n=54)\end{array}$ \\
\hline Can be used for open wounds & 4.1 & 4.2 \\
Assists with atraumatic dressing changes & 4.0 & 4.1 \\
Less pain with motion & 3.8 & 4.0 \\
No adverse effects & 3.8 & 4.1 \\
Assists with gaining motion & 3.7 & 3.4 \\
Additional cost & 3.7 & 3.7 \\
Decreased risk of infection & 3.6 & 4.4 \\
\hline
\end{tabular}

Note. $\mathrm{SiO}=$ silicone oil.

${ }^{a}$ Answers scored on a 5-point Likert scale; an overall "level of agreement" score of 0 indicates no agreement, with 5 indicating total agreement.

\section{Figure 3}

Reasons for No Longer Using Silicone Oil

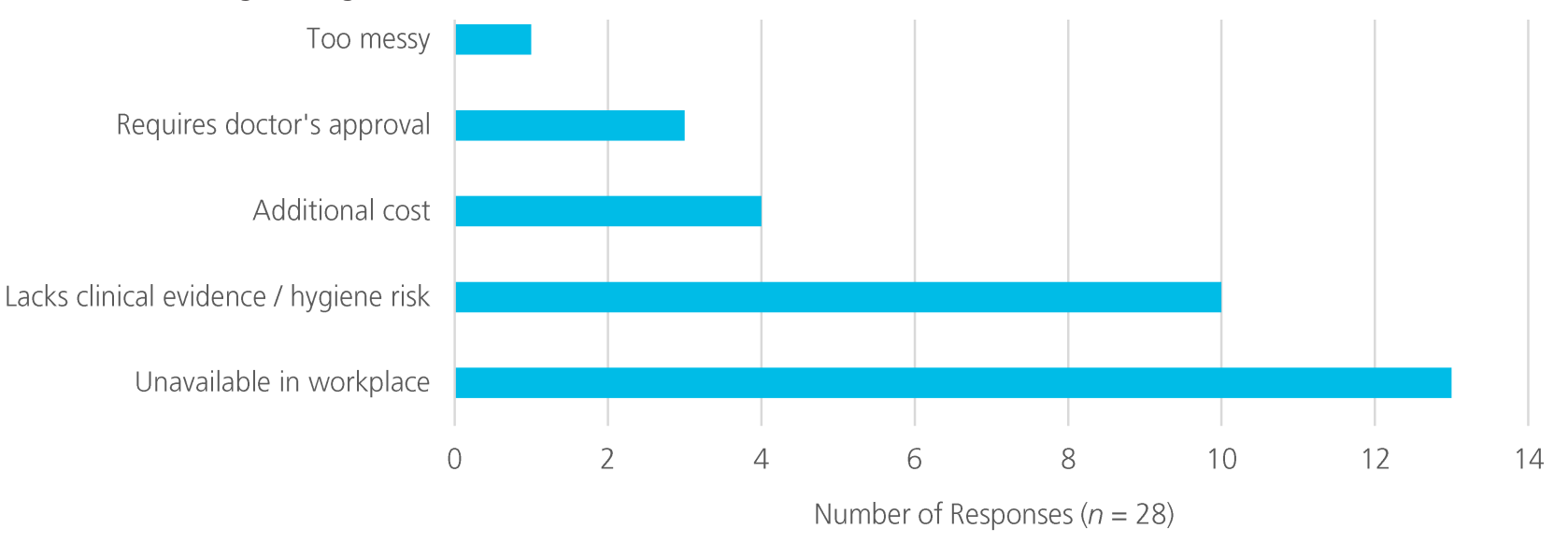




\section{Figure 4}

Reasons for Current Use of Silicone Oil

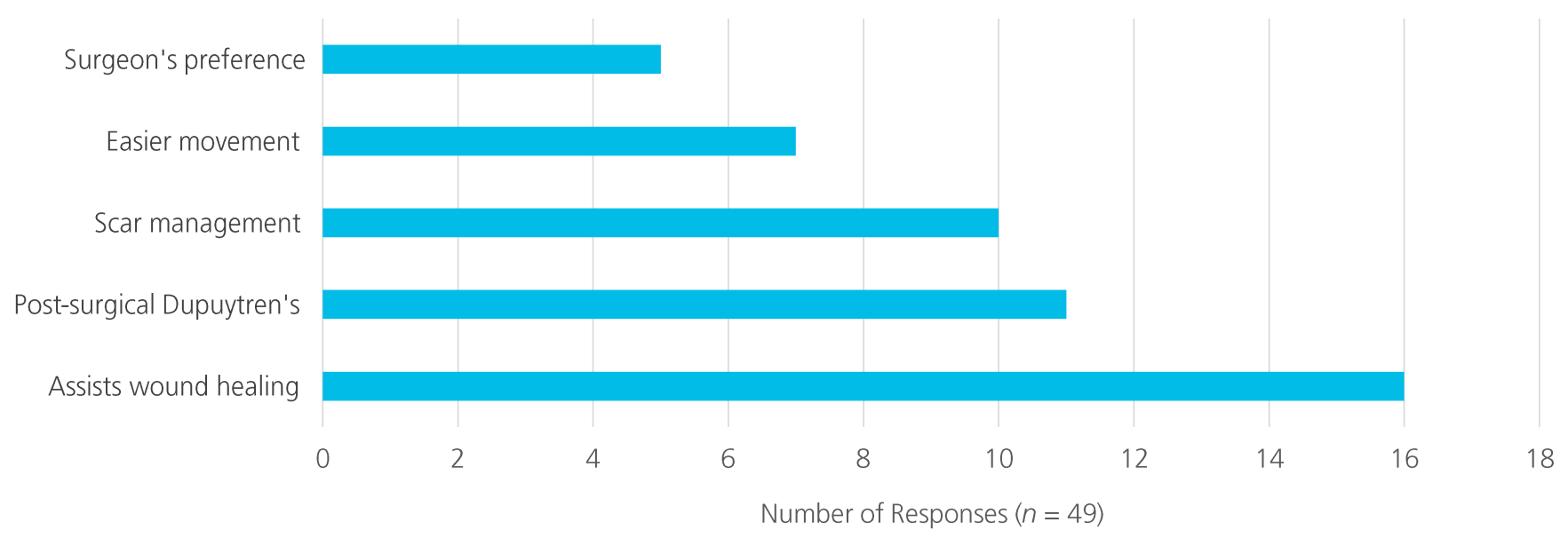

Dupuytren's rehabilitation. However, a third of therapists surveyed were unaware of SiO usage as an adjunct in hand rehabilitation.

The survey identified a range of clinical issues. Nonavailability of $\mathrm{SiO}$ at hospitals and clinics was one reason preventing therapists from incorporating $\mathrm{SiO}$ into rehabilitation programmes. Another factor impacting therapists' usage was that, at the time of administration of this questionnaire, SiO required a doctor's prescription. However, the New Zealand Government changed the medical classification of SiO to a medical device in 2014 (New Zealand Government, 2013), which means it can now be incorporated into a care plan by a therapist based upon clinical reasoning

From the results of this study, we can hypothesise that therapists with limited product knowledge are less likely to use SiO. However, this was not tested using statistical methods. Some respondents expressed concern regarding the risks of infection, cleanliness, and a lack of clinical evidence for SiO use. These concerns are valid as there is a paucity of literature in this area of hand therapy. Nevertheless, literature that does exist suggests that infection is not a concern. For example, the study of Thurston and McChesney (2002) examined SiO use for hand-wound rehabilitation and found that SiO did not support the growth of bacteria, despite the wound testing positive for bacterial growth.

Furthermore, while not in the field of hand research, there has been extensive investigation of $\mathrm{SiO}$ and infection in ophthalmic laboratory research. This body of work has identified strong antimicrobial properties associated with $\mathrm{SiO}$, particularly against staphylococcal pathogens (Arici et al., 2016; Chrapek et al., 2012; Ozdamar et al., 1999). Intraocular injections of antibiotics with and without $\mathrm{SiO}$ found treatment with $\mathrm{SiO}$ rapidly controlled infection and achieved better visual acuity when compared to intraocular injection of antibiotics alone (Bali et al., 2003). The research documenting antimicrobial and antiinflammatory properties of $\mathrm{SiO}$ is limited to ophthalmic research (Aras et al., 2001; Bali et al., 2003; Chrapek et al., 2012).
Conversely, therapists may be cognisant of literature detailing Swanson's silicone arthroplasty implants, used for finger joint replacements in arthritis (Bales et al., 2014; Swanson, 1972). Early publications reported complications with implanted silicone joint replacements, such as particulate synovitis, lymphadenopathy, and the formation of granuloma tissue (Christie et al., 1977; DeHeer et al., 1995; Kircher, 1980). However, Thurston (1997) reviewed 116 hand wounds mobilised in SiO over a 12-year period and did not find any of the adverse effects associated with implanted silicone such as granuloma, areas of inflammation or abnormal scar formation.

In contrast to the wide range of traumatic injuries and diagnoses associated with $\mathrm{SiO}$ usage in the earlier literature, the current study found post-surgical Dupuytren's commonly cited as the reason for SiO use. Hand therapists frequently see traumatic injuries, such as burns, de-gloving injuries, and tendon lacerations, and these share similar therapeutic goals to post-surgical Dupuytren's (Warwick, 2015), such as restoring motion with the least pain possible during wound healing. In the current study, therapists' perceptions regarding less pain with motion are consistent with previous authors who state, "The hand can be exercised with less pain than otherwise would occur" (Helal et al., 1982). Given this consistency, the rare citing of traumatic injuries as the reason for SiO use is surprising. The absence of published protocols that recommend $\mathrm{SiO}$ use for traumatic injuries may be a factor. Tendon repair rehabilitation, for example, is guided by very prescribed criteria, such as Kleinart (Hundozi et al 2013), Washington (Dovelle \& Heeter, 1989) and Saint John (Higgins \& Lalonde, 2016), but none suggest $\mathrm{SiO}$ use. In the absence of published protocols that specify SiO use, therapists may be reluctant to modify their current practice. Thurston (1997) details SiO use in post-surgical Dupuytren's, which may explain the use of SiO for this specific pathology.

Therapists in this study strongly agreed (4/5) on the utility of SiO in the daily replacement of wound dressings, where patients report less pain during dressing changes after SiO use. Spira and colleagues (1967) pioneered the use of SiO in plastic bags 
for the treatment of burned hands. The bag containing SiO acts as a form of dressing, while the sealed space within the bag allows unrestricted finger motion, which is advantageous during rehabilitation. Subsequently, silicone impregnated dressings were developed (Cutting et al., 2009; Platt et al., 1996). Silicone dressings adhere readily to intact skin, but do not stick to the moist wound surface. Subsequently, a lesser nociceptive stimulus occurs when the dressings are removed (White, 2005). Less pain and stress improve the speed of wound healing (Broadbent et al., 2003; Matsuzaki \& Upton, 2013; McGuire et al., 2006; Upton \& Solowiej, 2010), and these findings are consistent with therapists' perceptions that $\mathrm{SiO}$ usage heals hand wounds faster, with less pain experienced during range of motion exercises and atraumatic wound dressing changes.

The literature lacks studies on patient perceptions of SiO. Therefore, future studies should evaluate both the efficacy of $\mathrm{SiO}$ and qualitative investigation with the use of patient-rated outcome measures.

The strengths of this study include careful development of the questionnaire using Likert scales to ascertain therapists' opinions on $\mathrm{SiO}$, and the trial and refinement of the questionnaire before it was administered. The response rate achieved was acceptable (Richardson, 2005), although 60\% would have been desirable to reduce sample error and bias.

Study limitations include the use of a local regional sample for pilot testing and the absence of a working definition of $\mathrm{SiO}$ on the questionnaire. A clear definition of SiO may have eliminated possible respondent confusion. Seven respondents alluded to the point that SiO was helpful for scar management although it is silicone gel sheets that are explicitly designed and routinely used by therapists for scar care (Sawada \& Sone, 1990). Furthermore, the results of the study cannot be generalised beyond the conference attendees, and data collection was undertaken over seven years ago. Therefore, the views expressed are not representative of hand therapists worldwide.

\section{CONCLUSION}

This study found that $43 \%$ of therapists surveyed had used $\mathrm{SiO}$ as an adjunct in hand rehabilitation for the management of open hand wounds within New Zealand and Australia. Therapists surveyed agreed that SiO use permitted movement with less pain. Furthermore, therapists perceived that SiO use contributed to faster healing and atraumatic wound dressing changes.

The practical implications of this study include recognition of the low level of therapists' product knowledge and research relating to SiO. Therapists need easy access to recent evidence to support their clinical use of this adjunct. Surgical release of Dupuytren's hand joint contracture is a common surgery in New Zealand and a specific reason for clinical use of SiO. Addressing the nonavailability of $\mathrm{SiO}$ in the clinic could make this simple adjunct to hand rehabilitation more common in clinical practice where indicated.
This research highlights beliefs commonly held by New Zealand and Australian therapists regarding $\mathrm{SiO}$, and raises the profile of $\mathrm{SiO}$ as an adjunct therapy for hand wounds. Investigation of the effectiveness of SiO to improve wound healing, for example, in post-operative Dupuytren's contracture release wounds, is needed to improve clinical practice. Future research should first evaluate the feasibility and acceptability of treatment regimens, for example, using $\mathrm{SiO}$ in a home-based exercise programme, taking into account both clinicians' and patients' perspectives.

\section{KEY POINTS}

1. A quarter of hand therapists surveyed reported SiO use.

2. Therapists strongly agreed that SiO used in wound care permits movement with less pain.

3. Therapists agreed that SiO use impacted positively on wound dressing changes.

4. SiO is used specifically for post-surgical Dupuytren's rehabilitation.

\section{DISCLOSURES}

No funding was obtained for this study. There are no conflicts of interest which may be perceived to interfere with or bias this study.

\section{PERMISSIONS}

Ethical approval was obtained from the University of Otago Human Ethics Committee (reference number D13/346).

\section{ACKNOWLEDGEMENTS}

The authors acknowledge the cooperation of therapists attending the combined conference of Hand Therapy New Zealand and the Australia Hand Therapy Association held in Melbourne, Australia, in 2013.

\section{ADDRESS FOR CORRESPONDENCE}

Gail Donaldson, Centre for Health, Activity and Rehabilitation Research, (CHARR), School of Physiotherapy, University of Otago, PO Box 56, Dunedin, New Zealand.

Email: donga671@student.otago.ac.nz

\section{REFERENCES}

Aras, C., Ozdamar, A., Karacorlu, M., \& Ozkan, S. (2001). Silicone oil in the surgical treatment of endophthalmitis associated with retina detachment. International Ophthalmology, 24(3), 147-150. https://doi. org/10.1023/A:1021108907745

Arici, C., Aras, C., Tokman, H. B., \& Torun, M. M. (2016). An in vitro experimental study on the antimicrobial activity of silicone oil against anaerobic bacteria. Ocular Immunology and Inflammation, 24(2), 173-177. https://doi.org/10.3109/09273948.2014.971973

Bales, J. G., Wall, L. B., \& Stern, P. J. (2014). Long-term results of Swanson silicone arthroplasty for proximal interphalangeal joint osteoarthritis. The Journal of Hand Surgery, 39(3), 455-461. https://doi.org/https://doi. org/10.1016/j.jhsa.2013.11.008

Bali, E., Huyghe, P., Caspers, L., \& Libert, J. (2003). Vitrectomy and silicone oil in the treatment of acute endophthalmitis. Preliminary results. Bulletin de la Société Belge d'ophtalmologie, 288, 9-18. 
Braley, S. (1970). The chemistry and properties of the medical-grade silicones. Journal of Macromolecular Science Part A - Chemistry, 4(3), 529-544. https://doi.org/10.1080/00222337008074361

Broadbent, E., Petrie, K. J., Alley, P. G., \& Booth, R. J. (2003). Psychological stress impairs early wound repair following surgery. Psychosomatic Medicine, 65(5), 865-869. https://doi.org/10.1097/01. PSY.0000088589.92699.30

Chrapek, O., Vecerova, R., Koukalova, D., Maresova, K., Jirkova, B., Sin, M. \& Rehak, J. (2012). The in vitro antimicrobial activity of silicone oils used in ophthalmic surgery. Biomedical Papers of the Medical Faculty of the University Palacký, Olomouc, Czechoslovakia, 156(1), 7-13. https://doi. org/10.5507/bp.2011.060

Christie, A. J., Weinberger, K. A., \& Dietrich, M. (1977). Silicone lymphadenopathy and synovitis: Complications of silicone elastomer finger joint prostheses. JAMA, 237(14), 1463-1464. https://doi.org/10.1001/ jama.1977.03270410063025

Cutting, K., White, R., \& Hoekstra, H. (2009). Topical silver-impregnated dressings and the importance of the dressing technology. International Wound Journal, 6(5), 396-402. https://doi.org/10.1111/j.1742$481 \times .2009 .00635 . x$

Dave, V. P., Joseph, J., Jayabhasker, P., Pappuru, R. R., Pathengay, A., \& Das, T. (2019). Does ophthalmic-grade silicone oil possess antimicrobial properties? Journal of Ophthalmic Inflammation and Infection, 9, 20. https://doi.org/10.1186/s12348-019-0187-6

DeHeer, D. H., Owens, S. R., \& Swanson, A. B. (1995). The host response to silicone elastomer implants for small joint arthroplasty. The Journal of Hand Surgery, 20(3), S101-S109. https://doi.org/10.1016/S03635023(95)80180-4

Dovelle, S., \& Heeter, P. K. (1989). The Washington Regimen: Rehabilitation of the hand following flexor tendon injuries. Physical Therapy, 69(12), 1034-1040.

Helal, B., Chapman, R., Ellis, M., \& Gifford, D. (1982). The use of silicone oi for mobilisation of the hand. Journal of Bone and Joint Surgery (British), 64(1), 67-69. https://doi.org/10.1016/0007-1226(90)90189-7

Higgins, A., \& Lalonde, D. H. (2016). Flexor tendon repair post-operative rehabilitation: The Saint John protocol. Plastic and Reconstructive Surgery - Global Open, 4(11), e1134. https://doi.org/10.1097/ GOX.0000000000001134

Hundozi, H., Murtezani, A., Hysenaj, V., Hysenaj, V., \& Mustafa, A. (2013) Rehabilitation after surgery repair of flexor tendon injuries of the hand with Kleinert early passive mobilization protocol. Medical Archives, 67(2), 115-119. https://doi.org/10.5455/medarh.2013.67.115-119

Kircher, T. (1980). Silicone lymphadenopathy: A complication of silicone elastomer finger joint prostheses. Human Pathology, 11(3), 240-244. https://doi.org/10.1016/S0046-8177(80)80005-0

Kwan, P., Hori, K., Ding, J., \& Tredget, E. E. (2009). Scar and contracture: Biological principles. Hand Clinics, 25(4), 511-528. https://doi. org/10.1016/j.hcl.2009.06.007

Lazarus, G. S., Cooper, D. M., Knighton, D. R., Margolis, D. J., Percoraro, R. E., Rodeheaver, G., \& Robson, M. C. (1994). Definitions and guidelines for assessment of wounds and evaluation of healing. Wound Repair and Regeneration, 130(4), 489-493. https://doi.org/10.1001/ archderm.1994.01690040093015

Maciejczyk, S. (1961). Silicone fluid for burned hands. Polski Tygodnik Lekarski, 28, 1061-1065.

Matsuzaki, K., \& Upton, D. 2013). Wound treatment and pain management: A stressful time. International Wound Journal, 10(6), 638-644. https://doi. org/10.1111/j.1742-481X.2012.01038.x
McGuire, L., Heffner, K., Glaser, R., Needleman, B., Malarkey, W., Dickinson, S., Lemeshow, S., Cook, C., Muscarella, P., Melvin, W. S., Ellison, E. C., \& Kiecolt-Glaser, J. K. (2006). Pain and wound healing in surgical patients. Annals of Behavioral Medicine, 31(2), 165-172. https://doi.org/10.1207/ s15324796abm3102_8

New Zealand Government. (2013). Medicines Amendment Act 2013 (as at 03 June 2017). Ministry of Health. https://www.legislation.govt.nz/act/ public/2013/0141/latest/DLM4096106.html

Merritt, W. H. (1998). Written on behalf of the stiff finger. Journal of Hand Therapy, 11(2), 74-79. https://doi.org/10.1016/S0894-1130(98)80003-3

Norman, G. (2010). Likert scales, levels of measurement and the "laws" of statistics. Advances in Health Sciences Education, 15(5), 625-632. https:// doi.org/10.1007/s10459-010-9222-y

Ozdamar, A., Aras, C., Ozturk, R., Akin, E., Karacorlu, M., \& Ercikan, C. (1999). In vitro antimicrobial activity of silicone oil against endophthalmitis-causing agents. Retina, 19(2), 122-126. https://doi. org/10.1097/00006982-199902000-00006

Platt, A., Phipps, A., \& Judkins, K. (1996). A comparative study of silicone net dressing and paraffin gauze dressing in skin-grafted sites. Burns, 22(7), 543-545. https://doi.org/10.1016/0305-4179(96)00035-6

Richardson, J. T. (2005). Instruments for obtaining student feedback: A review of the literature. Assessment \& Evaluation in Higher Education, 30(4), 387-415. https://doi.org/10.1080/02602930500099193

Sawada, Y., \& Sone, K. (1990). Treatment of scars and keloids with a cream containing silicone oil. British Journal of Plastic Surgery, 43(6), 683-688. https://doi.org/10.1302/0301-620X.64B1.7068722

Spira, M., Miller, J., Hardy, S. B., \& Gerow, F. J. (1967). Silicone bag treatment of burned hands. Plastic and Reconstructive Surgery, 39(4), 357-365. https://doi.org/10.1097/00006534-196704000-00004

Swanson, A. B. (1972). Flexible implant resection arthroplasty. The Hand, 4(2), 119-134. https://doi.org/10.1016/0072-968X(72)90030-7

Thurston, A. J. (1997). Safety of silicone liquid in the post-operative management of Dupuytren's contracture. Australian and New Zealand Journal of Surgery, 67(6), 347-350. https://doi. org/10.1111/j.1445-2197.1997.tb01989.x

Thurston, A. J., \& McChesney, A. (2002). The bacterial colonisation of silicone oil used in the management of wounds of the hands - A source of nosocomial infection? Hand Surgery, 7(1), 21-26. https://doi.org/10.1142/ s0218810402000844

Upton, D., \& Solowiej, K. (2010). Pain and stress as contributors to delayed wound healing. Wound Practice \& Research, 18(3), 114-122.

Warwick, D. (Ed.). (2015). Dupuytrens disease: Federation of European Societies for Surgery of the Hand instructional course textbook. Edizioni Medico, Scientifiche.

Weeder, R. S., Brooks, H. W., \& Boyer, A. S. (1967). Silicone immersion in the care of burns. Plastic and Reconstructive Surgery, 39(3), 256-262. https:// doi.org/10.1097/00006534-196703000-00004

White, R. (2005). Evidence for atraumatic soft silicone wound dressing use. Wounds UK, 1(3), 104-109.

Yang, G., McGlinn, E. P., \& Chung, K. C. (2014). Management of the stiff finger: Evidence and outcomes. Clinics in Plastic Surgery, 41(3), 501-512. https://doi.org/10.1016/j.cps.2014.03.011 


\section{Appendix A}

\section{THE SILICONE OIL IN HAND REHABILITATION QUESTIONNAIRE}

This survey is being conducted by Gail Donaldson as part of her doctoral study being conducted at the University of Otago. This is a confidential survey which is being conducted to ascertain the level of usage and professional opinion of silicone oil held by New Zealand and Australian hand therapists.

Instructions: Please mark each question with a tick.

Provide answers when prompted and grade your responses as applicable.

1. Your professional registration status is:
$\square$ Physiotherapist
$\square$ Occupational therapist
$\square$ New Zealand registered
Australian registered

2. Your highest academic degree is:

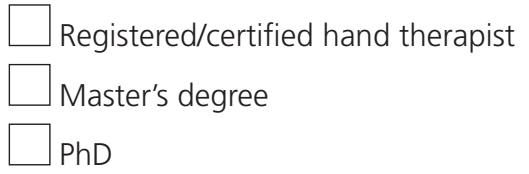

3. How many years of hand therapy experience do you have? years

4. The predominant area of your professional hand therapy practice is:
$\square$ Private practice
Public sector

5. Are you aware of the use of silicone oil in relation to hand therapy rehabilitation?

$\square$ Yes $\square$ No

6. Have you ever used silicone oil for your patients in hand rehabilitation?

$\square$ Yes $\square$ No

7. Do you currently use silicone oil for your patients in hand rehabilitation?

$\square$ Yes $\square$ No

8. If you currently use silicone oil, please state your reason for doing so.

9. If you do not currently use silicone oil or have done so in the past, please state your reason for not doing so now.
Please mark on the line the point that best describes your opinion regarding the therapeutic implications of silicone oil in the following statements.

10. Silicone oil immersion can be used in the presence of open wounds

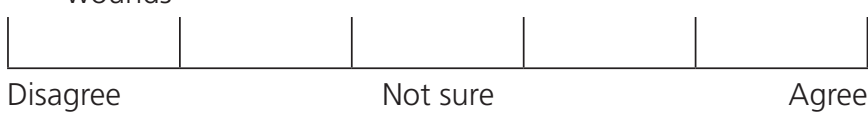

11. Silicone oil immersion helps improve range of motion of the hand

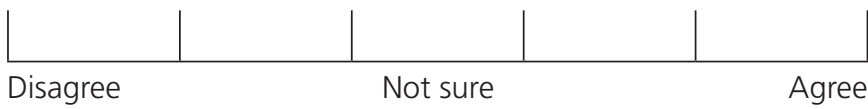

12. Silicone oil immersion allows movement with less pain

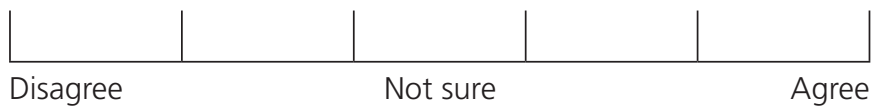

13. Silicone oil immersion assists with atraumatic dressing changes

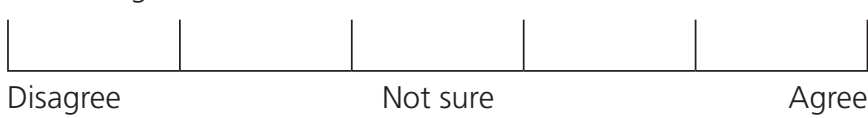

14. Silicone oil has adverse effects

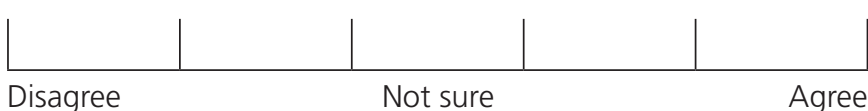

15. Silicone oil is costly

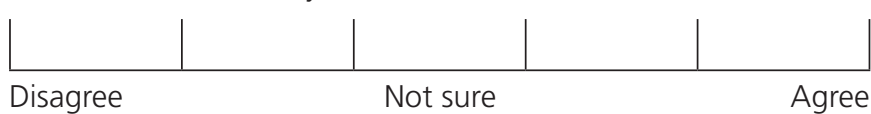

16. There is increased risk of infection with use of silicone oil

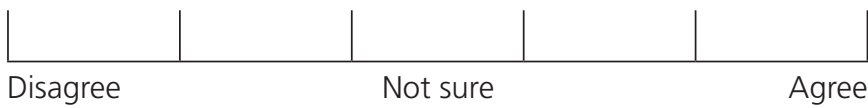

17. Do you want to add any additional comments about the perceived benefits of silicone oil in regard to hand rehabilitation?

18. Do you want to add any additional comments about the disadvantages of silicone oil? 